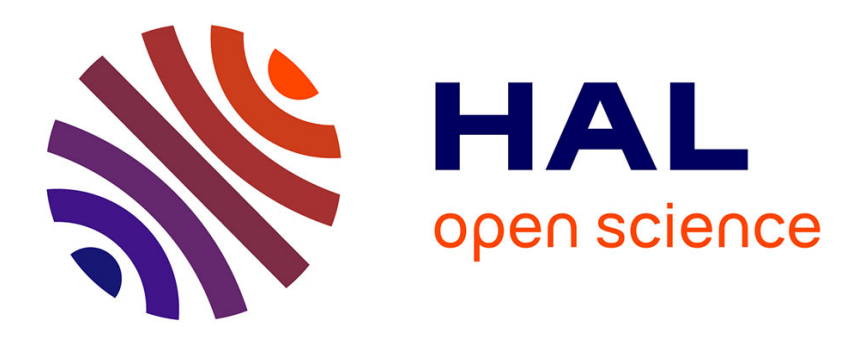

\title{
Quasi-stationary and ratio of expectations distributions: a comparative study
}

\author{
J.R. Artalejo, M.J. Lopez-Herrero
}

\section{To cite this version:}

J.R. Artalejo, M.J. Lopez-Herrero. Quasi-stationary and ratio of expectations distributions: a comparative study. Journal of Theoretical Biology, 2010, 266 (2), pp.264. 10.1016/j.jtbi.2010.06.030 . hal-00614182

\section{HAL Id: hal-00614182 \\ https://hal.science/hal-00614182}

Submitted on 10 Aug 2011

HAL is a multi-disciplinary open access archive for the deposit and dissemination of scientific research documents, whether they are published or not. The documents may come from teaching and research institutions in France or abroad, or from public or private research centers.
L'archive ouverte pluridisciplinaire HAL, est destinée au dépôt et à la diffusion de documents scientifiques de niveau recherche, publiés ou non, émanant des établissements d'enseignement et de recherche français ou étrangers, des laboratoires publics ou privés. 


\section{Author's Accepted Manuscript}

Quasi-stationary and ratio of expectations distributions: a comparative study

J.R. Artalejo, M.J. Lopez-Herrero

PII: S0022-5193(10)00320-6

DOI: doi:10.1016/j.jtbi.2010.06.030

Reference: YJTBI 6050

To appear in: $\quad$ Journal of Theoretical Biology

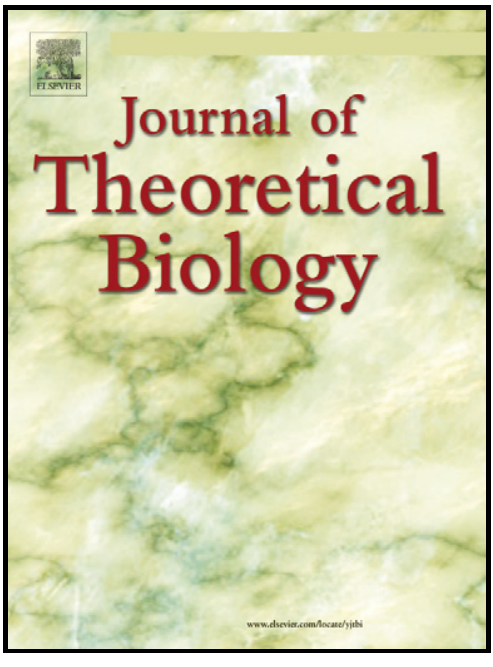

www.elsevier.com/locate/yjtbi

Received date: $\quad 24$ November 2009

Revised date: 22 June 2010

Accepted date: 22 June 2010

Cite this article as: J.R. Artalejo and M.J. Lopez-Herrero, Quasi-stationary and ratio of expectations distributions: a comparative study, Journal of Theoretical Biology, doi:10.1016/j.jtbi.2010.06.030

This is a PDF file of an unedited manuscript that has been accepted for publication. As a service to our customers we are providing this early version of the manuscript. The manuscript will undergo copyediting, typesetting, and review of the resulting galley proof before it is published in its final citable form. Please note that during the production process errors may be discovered which could affect the content, and all legal disclaimers that apply to the journal pertain. 


\author{
Quasi-stationary and ratio of expectations \\ distributions: A comparative study \\ J.R. Artalejo ${ }^{a, 1}$, M.J. Lopez-Herrero ${ }^{2}$ \\ ${ }^{a, 1}$ Corresponding author \\ Department of Statistics and Operations Research \\ Faculty of Mathematics \\ Complutense University of Madrid \\ 28040 Madrid, Spain \\ Email: jesus_artalejo@mat.ucm.es \\ ${ }^{2}$ School of Statistics \\ Complutense University of Madrid \\ 28040 Madrid, Spain
}

Email: lherrero@estad.ucm.es

\begin{abstract}
Many stochastic systems, including biological applications, use Markov chains in which there is a set of absorbing states. It is then needed to consider analogues of the stationary distribution of an irreducible chain. In this context, quasi-stationary distributions play a fundamental role to describe the long-term behavior of the system. The rationale for using quasi-stationary distribution is well established in the abundant existing literature. The aim of this study is to reformulate the ratio of means approach (Darroch and Seneta, $1965,1967)$ which provides a simple alternative. We have a two-fold objective. The first objective is viewing quasi-stationarity and ratio of expectations as two different approaches for understanding the dynamics of the system before absorption. At this point, we remark that the quasi-stationary distribution and a ratio of means distribution may give or not give similar information. In this way, we arrive to the second objective; namely, to investigate the possibility of using the ratio of expectations distribution as an approximation to the quasistationary distribution. This second objective is explored by comparing both distributions in some selected scenarios, which are mainly inspired in stochastic epidemic models. Previously, the rate of convergence to the quasi-stationary regime is taking into account in order to make meaningful the comparison.
\end{abstract}

\title{
1. Introduction
}

There exists a vast literature that uses Markov chains to model a biological population. A good reference to find the underlying mathematical theory is the textbook by Allen (2003) and its references. As examples of recent applications of continuous-time Markov chains in population biology, we mention some papers published in this journal (Casagrandi and Gatto, 2006; Fouchet et al., 2008; Stirk et al., 2008; Xu et al., 2007).

Stochastic biological models based on Markov chains typically consist of a set $S_{T}$ of transient states where the process evolves until it escapes to a set 
of absorbing states $S_{A}$. In many applications $S_{A}$ reduces to the sole state 0 . Due to the reducible character of the Markov chain, the stationary distribution becomes degenerate in the sense that it only assigns probability mass to the absorbing states. This fact motivates the following fundamental problem:

There exist a need for probabilistic measures of the system behavior before absorption. Analogues of the stationary distribution of the irreducible case should be considered and compared.

The above description of the problem under study reflects the spirit of the seminal work by Darroch and Seneta $(1965,1967)$ who suggested several possibilities including quasi-stationary distributions and ratio of means.

The term quasi-stationarity refers to the distribution of the Markov chain by conditioning on the event that absorption has not occurred yet. Let us assume that the Markov chain starts with the initial distribution on the transient states $\left\{u_{i} ; i \in S_{T}\right\}$. Then, the distribution $\left\{u_{i} ; i \in S_{T}\right\}$ is called a quasistationary distribution when the distribution of the Markov chain, conditional on it has not yet absorbed at time $t$, remains equal to $\left\{u_{i} ; i \in S_{T}\right\}$ over time. On the other hand, the quasi-stationary distribution can also be presented as a limiting conditional probability (Darroch and Seneta, 1967; Pollett, 2001). This interpretation motivates the significant role of the quasi-stationarity as a measure of the time spent by the process in the transient states when the time to absorption is sufficiently long. For a formal definition of quasi-stationarity, we refer the reader to Section 2.

If the transient set $S_{T}$ is finite and irreducible, then the existence of the quasi-stationary distribution is guaranteed. However, even in the case of birth and death processes, it is typically impossible to evaluate explicitly the quasistationary distribution. One is therefore lead to consider iterative methods, approximations and asymptotic results for the quasi-stationary distribution. Many satisfactory approximate results have been obtained, see Nåsell's papers (1996, 1999a, 2001) and their references. There are important models in population biology where the transient set $S_{T}$ is reducible. For a comprehensive treatment of this case, see van Doorn and Pollett (2008). Roughly speaking, one could say that the quasi-stationary distribution, if it exists, gives positive probability to a certain subset $S_{T^{+}}$of $S_{T}$ but the quasi-stationary probability of a state $i \in S_{T}-S_{T^{+}}$is zero. This is a striking fact which merits some extra attention. An example of this situation is given by the $S I R$ epidemic model, see Subsection 3.2.1. If $S_{T}$ is infinite, the analysis is essentially more complicated. Conditions for the existence of the quasi-stationary distribution are mathematically involved. An attempt to summarize conditions given in terms of the infinitesimal transition rates can be found in Pollett (2001). The book by Seneta (1981) is a basic reference for the general mathematical theory.

The above discussion on the concept of quasi-stationarity, which will be extended in Section 2, gave the authors an initial motivation to retrieve the $R E$-distribution (ratio of expectations distribution) as another natural measure of the behavior of absorbing Markov chains before absorption occurs. Given the initial state, the $R E$-distribution will be defined as a ratio between the time that 
the process spends at each transient state and the expected time to absorption. In the sequel, we will show how the $R E$-distribution has a meaningful interpretation. Moreover, it exists and can be evaluated with a great generality. The idea of presenting a ratio of means as an analogue of the stationary distribution of an irreducible Markov chain was suggested in the early work by Ewens (1963) and Darroch and Seneta $(1965,1967)$.

One of the fundamental reasons to study biological models is to provide tools for getting insight of the underlying biological reality. Different models and approaches can be considered, but the final objective is always to capture the essential features of a biological system. It would be difficult and subjective to decide which approach is preferable. In fact, stochastic models are based on the concept of probability, whose basic roots are indeed subject to different interpretations (see e.g. the different methods for quantifying uncertainty or the different concepts of convergence of random variables). The existence of several methods and approaches should be interpreted as an advantage rather than as a problem. This open spirit inspires our comparative study in the paper. It is clear that the quasi-stationarity is a well established concept and there is no doubt about its usefulness. Our aim is to rescue the ratio of expectations approach and show that it is worthy of some extra attention. The comparison between the quasi-stationary distribution and the $R E$-distribution is justified only if the convergence to quasi-stationarity is relatively fast. If this is the case, the comparative analysis done in the sequel may lead to the conclusion that both approaches give similar or different information. As a result, one may see pros and cons. However, it is so far of our intention to suggest the possible superiority of one approach over the other. The existence of different alternatives to measure the system behavior before absorption enriches our knowledge and provides complementary insight of the underlying biological problem.

The rest of the paper is organized as follows. In Section 2, the $R E$-distribution is introduced in the context of a general absorbing continuous-time Markov chain. Some emphasis is done on a general comparison with respect to the quasi-stationary distribution. Section 3 explores applications to stochastic population models. More specifically, we first consider the irreducible unidimensional case and deal with a class of birth and death processes including the stochastic SIS epidemic model (Subsection 3.1.1) and a linear growth model (Subsection 3.1.2). The SIR epidemic model (Subsection 3.2.1) provides a nice example to deal with the reducible case. As an example of a bidimensional tractable case where $S_{T}$ is irreducible, we consider a finite $S I R$ model with demography (Subsection 3.2.2). These models provide appropriate scenarios to illustrate the tractability and interest of the $R E$-distribution as a measure of the system behavior before absorption. Moreover, a comparison with results based on the quasi-stationary distribution is performed. Our comparative study includes some theoretical justifications and discussion on the related computational issues. Finally, in the discussion section (Section 4), the main features of the quasi-stationary distribution and the $R E$-distribution are recapitulated. 
The discrete-time case is also briefly outlined.

\section{Comparing quasi-stationarity and the $R E$-distribution}

We shall employ an absorbing continuous-time Markov chain $\{X(t) ; t \geq 0\}$ with countable state space $S$ to model a biological population. Suppose that $S_{A}$ denotes the set of absorbing states and $S_{T}$ is the set of transient states, so $S=S_{A} \cup S_{T}$. Let $Q=\left[q_{i j}\right]$ be the infinitesimal generator of the Markov chain. Then, $q_{i}=-q_{i i}$ represents the rate of the exponential sojourn time at state $i$. For states $i \in S_{A}$, we define $q_{i}=0$.

The quasi-stationary distributions have been widely used to measure the distribution of the process given that the absorption has no yet taken place. Thus, the starting point is the conditional probability

$$
u_{i}(t)=P\{X(t)=i \mid T>t\}, i \in S_{T},
$$

where $T=\sup \left\{t \geq 0: X(t) \in S_{T}\right\}$ denotes the absorption time.

Now suppose the Markov chain starts with the initial distribution $a_{i}=$ $P\{X(0)=i\}$, for $i \in S_{T}$. If there exists a starting distribution $a_{i}=u_{i}$ such that

$$
P\{X(t)=i \mid T>t\}=u_{i}, i \in S_{T},
$$

for all $t \geq 0$, then $\left\{u_{i} ; i \in S_{T}\right\}$ is called a quasi-stationary distribution.

From definition (2.1), it is clear that the quasi-stationary distribution is independent of $t$ if the Markov chain starts with initial distribution $\left\{u_{i} ; i \in S_{T}\right\}$.

Let us first assume that $S_{T}$ is finite and irreducible. Then, it is wellknown (Darroch and Seneta, 1967) that the quasi-stationary distribution is a left eigenvector corresponding to the eigenvalue with maximal real part of the sub-generator $Q_{S_{T}}$ associated to the transient states. We denote such maximal eigenvalue as $-\alpha$. It is well-known that $\alpha$ is real and positive. This result provides a first option for numerical computations.

There also exists a limiting interpretation of the quasi-stationary distribution which states that

$$
\lim _{t \rightarrow \infty} P\{X(t)=i \mid T>t\}=u_{i}, i \in S_{T},
$$

independently of the initial distribution $\left\{a_{i} ; i \in S_{T}\right\}$.

The limiting result (2.2) supports the accepted idea that the quasi-stationary distribution provides a good measure of the system dynamics before absorption, but restricting only to those realizations in which the time to absorption is sufficiently large. Another doubly-limiting approach to quasi-stationarity has also been considered in the literature, see for instance Darroch and Seneta (1967) and Pollett (2001).

There is no doubt about the useful information provided by the quasistationary distribution in the case where the Markov chain is known to be in the set $S_{T}$ after a very long time. However, there exists a second scenario in which the quasi-stationary distribution is also helpful to describe the behavior of the system before absorption. This is the case where the process reaches the 
quasi-stationary regime after a relatively short time and, after a substantially longer period, absorption will occur.

At this point, a rule to identify the second scenario in practice is needed. A natural solution is given in terms of a comparison between the rate of convergence to absorption and the rate of convergence to the quasi-stationary distribution. The former is given by $\alpha$, while the latter is $\alpha^{\prime}-\alpha$ where $-\alpha^{\prime}$ is the real part of the eigenvalue with the next smallest real part, see formulas (1.1) and (3.3) in Darroch and Seneta (1967). As a result, the quasi-stationary distribution should be used to describe the behavior before absorption only if $R_{u}=2 \alpha / \alpha^{\prime}<1$. Then, the comparison between the quasi-stationary distribution and the $R E$-distribution becomes meaningful.

It is well reported in the literature (Nåsell, 2001) that an analytical solution to the quasi-stationary probabilities only exists in a few special cases. Among them, we mention some birth and death processes where the birth and death rates are either constants or linear functions of the state $i \in S_{T}$. As a result, approximations and numerical methods of computation are required.

If we focus on finite birth and death processes with absorbing state 0 , then the difficulties to obtain an analytical solution are related to the non-linear form of the system of equations governing the quasi-stationary probabilities. However, one could use efficient iteration methods (Cavender, 1978; Nåsell, 2001), approximations and asymptotic results. Kryscio and Lefèvre (1989) summarized the first work on two popular approximations. The first one, denoted by $p^{(0)}$, approximates the original birth and death process by assuming that $\mu_{1}=0$; that is, the death rate of a population of size 1 is equal to 0 which amounts the original process with the origin 0 removed. In the context of the $S I S$ epidemic model a second approximation, called $p^{(1)}$, is obtained by assuming the existence of one permanently infected individual. The two approximations are birth and death processes without absorbing states, so their stationary distributions can be used to approximate the quasi-stationary distribution. In epidemiological models, the goodness of the approximation depends on the so-called transmission factor, which is denoted by $R_{0}$. The transmission factor is interpreted in biological terms as the average number of contacts produced by one individual during the period of infectivity, when the individual is introduced into a completely susceptible population (Allen, 2003). The literature for the SIS model is particularly rich (see Subsection 3.1.1), but there also exists an important number of papers dealing with approximations and asymptotic results for other stochastic epidemic models (e.g. the Verhulst logistic model (Nåsell, 2001), the $S I R$ model with demography (Nåsell 1999b, 2001; van Herwaarden and Grasman, 1995, etc.)).

We now concentrate on the case where $S_{T}$ is finite but reducible. This case has been investigated in a recent paper by van Doorn and Pollett (2008). Some preliminary notation is needed. Suppose that $S_{T}$ consists of $L$ communicating classes $S_{k}$, for $1 \leq k \leq L$. A partial order on $\left\{S_{k} ; 1 \leq k \leq L\right\}$ is defined by writing $S_{i} \prec S_{j}$ when class $S_{i}$ is accessible from $S_{j}$. Let $-\alpha_{k}$ be the (negative) eigenvalue with maximal real part of the sub-generator $Q_{k}$ corresponding to the states in $S_{k}$. Then, the eigenvalue of $Q_{S_{T}}$ with maximal real part is obtained 
as $-\alpha$, where $\alpha=\min _{1 \leq k \leq L} \alpha_{k}$. We also define $I(\alpha)=\left\{k: \alpha_{k}=\alpha\right\}$ and $a(\alpha)=\min I(\alpha)$.

We can now summarize the main results in van Doorn and Pollett (2008). If $-\alpha$ has a geometric multiplicity one, then the Markov chain has a unique quasi-stationary distribution $\left\{u_{i} ; i \in S_{T}\right\}$ from which $S_{a(\alpha)}$ is accessible. The $j$ th component of $\left\{u_{i} ; i \in S_{T}\right\}$ is positive (i.e., $j \in S_{T^{+}}$) if and only if state $j$ is accessible from $S_{a(\alpha)}$. A simple necessary and sufficient condition for establishing that $-\alpha$ has geometric multiplicity one is that $\left\{S_{k} ; k \in I(\alpha)\right\}$ is linearly ordered, that is, $S_{i} \prec S_{j} \Longleftrightarrow i \leq j$, for all $i, j \in I(\alpha)$.

Following an example given by van Doorn and Pollett (2008), we notice that the quasi-stationary distribution of the pure death process on $S=\{0,1,2\}$ with absorbing state 0 and $\mu_{i}>0$, for $i \in S_{T}=\{1,2\}$, is given by

$$
\left(u_{1}, u_{2}\right)= \begin{cases}(1,0), & \text { if } \mu_{1} \leq \mu_{2} \\ \left(\frac{\mu_{2}}{\mu_{1}}, 1-\frac{\mu_{2}}{\mu_{1}}\right), & \text { if } \mu_{2}<\mu_{1}\end{cases}
$$

Suppose that $X(0)=2$ and $\mu_{1}=\mu_{2}=\mu$, then formula (2.3) says that the process will be in state $i=1$ with probability 1 , if after a long time absorption has not yet occurred. In other words, the set of states having positive mass is reduced to $S_{T^{+}}=\{1\}$. However, the absorption certainly occurs as far as $t \rightarrow \infty$, and the expected amount of time that the processes spends in state $i \in S_{T}=\{1,2\}$ is $1 / \mu$, given that $X(0)=2$. Thus, one could appeal to a natural frequency interpretation of the concept of probability to conclude that the even distribution that gives mass 0.5 to each state $i \in S_{T}=\{1,2\}$ provides the natural measure of the process behavior at an arbitrary time before absorption. However, if one wishes to know the state distribution after a very long time, given that the process is known to be in $S_{T}$, then the quasi-stationary distribution is the natural solution.

Comparing the dynamics of the pure death model versus most epidemic models, one may conclude that they are so different. It is certainly true, but our interest in the pure death model is just that it provides an extremely simple scenario where the quasi-stationary distribution and the $R E$-distribution exhibit drastically different solutions. The first, but not the unique, key to understand this peculiar fact is that $S_{T}$ is reducible.

The concept of quasi-stationarity becomes more complicated when $S_{T}$ is infinite. Then, a natural question of interest is to find finite approximations. Let us assume that $S_{T}$ is irreducible. Then, under appropriate conditions (Breyer and Hart, 2000) there exists an increasing sequence of finite irreducible truncations whose quasi-stationary distributions are used to approximate the corresponding one of the original infinite process. The parallel discrete-time results can be found in Seneta (1981).

We now turn our attention to the reformulation of the $R E$-distribution. Let $T_{j}$ be the time that process $\{X(t) ; t \geq 0\}$ spends in state $j \in S_{T}$ before absorption. Then, given that $X(0)=i \in S_{T}$, we define 


$$
P_{i}(j)=\frac{E_{i}\left[T_{j}\right]}{E_{i}[T]}, i, j \in S_{T} .
$$

In the light of (2.4), we notice that the $R E$-distribution always exists whether $S_{T}$ is finite or infinite, provided that the expected time to absorption $E_{i}[T]=$ $E[T \mid X(0)=i]$ is finite. Moreover, it should be pointed out that the $R E$ distribution assigns positive probability to all state $j$ accessible from the initial state $i$. Compared to the quasi-stationary distribution, this is an important difference.

The $R E$-distribution has a meaningful probabilistic interpretation. Let us construct the ideal replicated model obtained by assuming that at each extinction the biological model restarts in the same initial state $i \in S_{T}$. This replicated model is a regenerative process so its limiting distribution equals the expected amount of time the process spends in the state $j$ during one regeneration cycle divided by the expected length of one cycle, see Theorem 8.26 in Kulkarni (1995). In Subsection 3.1.1, we will explore in more detail the relationship between the quasi-stationary and ratio of expectation distributions, and the model that immediately restarts in state $i$.

The ratio of means distribution studied in the early literature is now obtained as the following unconditional version of the $R E$-distribution (2.4):

$$
P(j)=\frac{\sum_{i \in S_{T}} a_{i} E_{i}\left[T_{j}\right]}{\sum_{i \in S_{T}} a_{i} E_{i}[T]}, j \in S_{T} .
$$

In the next section we deal with a variant of $P(j)$ in (2.5) obtained by replacing the initial distribution $\left\{a_{i} ; i \in S_{T}\right\}$ by weights $\left\{\omega_{i} ; i \in S_{T}\right\}$, which do not preserve the interpretation as initial probabilities at time $t=0$.

Darroch and Seneta $(1965,1967)$ do not pursue the use of $\left\{P(j) ; j \in S_{T}\right\}$ further because it depends on the initial distribution $\left\{a_{i} ; i \in S_{T}\right\}$. More concretely, they clearly stated that the reason for the dependence of the ratio of means on the initial distribution is that the absorption time is not long enough for the dependence on the initial distribution to wear off. Darroch and Seneta (1965, Section 4) also explain that the quasi-stationary distributions do not depend on the initial distribution and are derived, roughly speaking, by considering only those realizations in which the absorption time is long. Since then, the ratio of means distribution has been largely ignored.

There is no doubt that the dependence on the initial distribution is a significant conceptual difference between both approaches. In population biology, it is often known that a certain population has been evolving for a long time, and that it has not reached the extinction yet. Furthermore, it may be very difficult, or even impossible, to know the exact initial distribution. From this perspective, the quasi-stationary distribution gives a very satisfactory approximation of the system state before absorption (i.e., the first objective mentioned in the abstract). However, we think that, whenever it can be possible, the effect of the initial distribution should be taken into account. We note that formula 
(2.4) allows the use of the $R E$-distribution not only when the time to absorption is large (i.e., the region $R_{0}>1$ in epidemic models) but also in cases where absorption is reached soon. If the absorption time is not long enough, then the initial distribution influences the current system state. In this case, the efforts to deal with the $R E$-distribution are welcome. For example, an outbreak of head lice (pediculosis capitis) in a school should end within a not so long time horizon, and the school administrators should have some knowledge about the number of pupils infected when the outbreak was detected. Despite of whether it can be observed or not, it seems reasonable to assume that an outbreak of many epidemics starts with a single infective who introduces the infection into the population. Bayesian methods (Clancy and O'Neill, 2008) and the maximum entropy principle can be helpful for estimating initial distributions. On the other hand, in those situations where the system parameters lead to long absorption times, the effect of the initial state $X(0)=i$ is weak. The numerical examples in Section 3 illustrate that the quasi-stationary distribution and the $R E$-distribution are in fact close when the absorption time is long. In this important case, the $R E$-distribution can be used as a simple approximation to the quasi-stationary distribution (i.e., the second objective in this paper).

To conclude this section, we next discuss how to compute the $R E$-distribution. Obviously, $E_{i}[T]=\infty$ if $P\{T<\infty \mid X(0)=i\}<1$. In the case $P\{T<\infty$ $\mid X(0)=i\}=1$ (i.e., absorption occurs with probability 1 ), the expectations $\left\{E_{i}[T] ; i \in S_{T}\right\}$ are given by the smallest non-negative solution to the system of linear equations (see Theorem 6.19 in Kulkarni, 1995)

$$
E_{i}[T]=\frac{1}{q_{i}}+\sum_{\substack{j \in S_{T} \\ j \neq i}} \frac{q_{i j}}{q_{i}} E_{j}[T], i \in S_{T} .
$$

Then, by expressing (2.6) in matrix form, we obtain

$$
Q_{S_{T}} \mathbf{m}=-\mathbf{e}
$$

where $\mathbf{m}$ and $\mathbf{e}$ are column vectors with dimension the cardinality of the subset $S_{T}$. The entries of $\mathbf{m}$ are $E_{i}[T]$, whereas all entries of $\mathbf{e}$ are all equal to 1 .

For a fixed $j \in S_{T}$, we employ a first-step argument to find that

$$
E_{i}\left[T_{j}\right]=\frac{\delta_{i j}}{q_{i}}+\sum_{\substack{k \in S_{T} \\ k \neq i}} \frac{q_{i k}}{q_{i}} E_{k}\left[T_{j}\right], i \in S_{T},
$$

where $\delta_{i j}$ denotes Kronecker's delta which is defined as follows:

$$
\delta_{i j}=\left\{\begin{array}{lc}
1, & \text { if } i=j \\
0, & \text { otherwise }
\end{array}\right.
$$

Thus, the matrix form version of equation (2.7) is

$$
Q_{S_{T}} \mathbf{m}_{j}=-\mathbf{e}_{j}
$$


where $\mathbf{m}_{j}$ is now the column vector with entries $E_{i}\left[T_{j}\right]$, for $i \in S_{T}$, and $\mathbf{e}_{j}$ is a column vector such that all entries are equal to 0 , except the $j$ th which is equal to 1 .

\section{Applications to stochastic population models}

In Section 2 we introduced the $R E$-distribution in the context of an absorbing continuous-time Markov chain with countable state space. In this section we provide applications to some selected population models: the classical $S I S$ epidemic model, a linear growth model, the classical $S I R$ model and a finite $S I R$ model with demography. A comparison with the quasi-stationary distribution is done. Our results include a few theoretical findings and numerical experiments. The main results for the classical $S I S$ and $S I R$ epidemic models can be found in several textbooks (Andersson and Britton, 2000; Allen, 2003; Bailey, 1990; Daley and Gani, 1999). For discussions about variants and generalizations, please consult some recent papers (Allen, 2008; Clancy, 2005; Fouchet et al., 2008; Lindholm, 2008; Xu et al., 2007) and the references therein.

\subsection{General birth and death processes}

We consider a birth and death process $\{X(t) ; t \geq 0\}$ with state space $S=$ $\{0,1, \ldots, N\}$. Both the finite case $(N<\infty)$ and the case of infinite state space $(N=\infty)$ can be studied. The infinitesimal transition rate from state $i$ to state $i+1$ is denoted by $\lambda_{i}>0$ (birth rate), for $1 \leq i \leq N-1$, whereas the transition rate from state $i$ to state $i-1$, for $1 \leq i \leq N$, is denoted by $\mu_{i}>0$ (death rate). We assume that the origin is an absorbing state, so $\lambda_{0}=0$. Moreover, if $N<\infty$ then we take $\lambda_{N}=0$ to be consistent with the assumption that $S$ is finite. Figure 1 shows the state space and transitions.

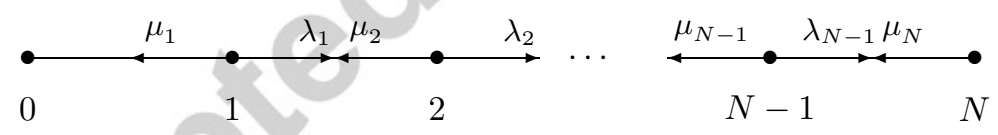

Fig. 1. States and transitions of the birth and death model

For each fixed $j \in\{1, \ldots, N\}$, equation (2.7) yields

$$
\begin{gathered}
E_{0}\left[T_{j}\right]=0, \\
\left(\lambda_{i}+\mu_{i}\right) E_{i}\left[T_{j}\right]=\mu_{i} E_{i-1}\left[T_{j}\right]+\lambda_{i} E_{i+1}\left[T_{j}\right], i \neq j, 1 \leq i \leq N, \\
\left(\lambda_{j}+\mu_{j}\right) E_{j}\left[T_{j}\right]=\mu_{j} E_{j-1}\left[T_{j}\right]+\lambda_{j} E_{j+1}\left[T_{j}\right]+1 .
\end{gathered}
$$

Using methods of finite difference equations, we find that the solution of the system (3.1)-(3.3) is given by

$$
E_{i}\left[T_{j}\right]=\frac{1}{\mu_{j}} \sum_{k=1}^{\min (i, j)} \prod_{n=k}^{j-1} \frac{\lambda_{n}}{\mu_{n}}, 1 \leq i, j \leq N
$$


Since $(T \mid X(0)=i)=\sum_{j=1}^{N}\left(T_{j} \mid X(0)=i\right)$, summing $(3.4)$ over $j$ we obtain

$$
P_{i}(j)=\frac{E_{i}\left[T_{j}\right]}{E_{i}[T]}=\frac{\frac{1}{\mu_{j}} \sum_{k=1}^{\min (i, j)} \prod_{n=k}^{j-1} \frac{\lambda_{n}}{\mu_{n}}}{\sum_{j=1}^{N} \frac{1}{\mu_{j}} \sum_{k=1}^{\min (i, j)} \prod_{n=k}^{j-1} \frac{\lambda_{n}}{\mu_{n}}}, 1 \leq i, j \leq N .
$$

We emphasize that explicit formula (3.5) for the $R E$-distribution applies both for $N<\infty$ and $N=\infty$. Furthermore, after simple algebraic manipulations, it is easy to observe that the expression given in the denominator for $E_{i}[T]$ is in agreement with the well-known expression for the expected time until extinction, see formula (5.7) in Norden (1982) and formula (6.22) in Allen (2003).

We conclude this subsection by noticing that the whole probability distribution of the variables $T_{j} \mid X(0)=i$ can be easily determined in terms of a system of linear equations for the Laplace transforms $E\left[\exp \left\{-s T_{j}\right\} \mid X(0)=i\right]$. Once the Laplace transforms have been computed, the corresponding density functions, or the survival probabilities $P_{i}\left\{T_{j}>x\right\}$, can be obtained by numerical inversion methods (Cohen, 2007). Equations for higher moments $E_{i}\left[T_{j}^{k}\right]$ follow by direct differentiation of the Laplace equations.

\subsubsection{The classical SIS model}

The general formulation given in Subsection 3.1 includes as particular cases many stochastic population models. In particular, for the case $N<\infty$, we mention the stochastic version of the Verhulst logistic model and the stochastic SIS epidemic model (Allen, 2003). The latter model corresponds to the following specification:

$$
\begin{gathered}
\lambda_{i}=\frac{\beta}{N} i(N-i), 0 \leq i \leq N \\
\mu_{i}=(\gamma+b) i, \quad 1 \leq i \leq N
\end{gathered}
$$

where $\beta, \gamma$ and $b$ respectively denote the contact, recovery and birth-death rates. We assume that $b=0$. Then, the transmission factor is $R_{0}=\beta / \gamma$.

The quasi-stationary distribution of the $S I S$ model cannot be obtained explicitly. However, the probabilities $\left\{u_{i} ; 1 \leq i \leq N\right\}$ can be computed by using the recursive procedure proposed by Cavender (1978). Two methods for implementing the recursion are summarized by Nåsell (2001). In addition, the two approximations $p^{(0)}$ and $p^{(1)}$ described in Section 2 are often useful. The quality of the approximations is discussed with respect to the three parameter regions where $R_{0}>1\left(R_{0}\right.$ is distinctly larger than 1$), R_{0}<1$ ( $R_{0}$ is distinctly smaller than 1 ) and $R_{0}$ is close to 1 (transient region), as $N \rightarrow \infty$. Following Nåsell (1999a), we notice that in the regions $R_{0}>1$ and $R_{0}<1$ the quasistationary distribution is well approximated by $p^{(0)}$ and $p^{(1)}$, respectively. Nåsell 
(1999a) derives a more sophisticated approximation for the intricate transient case. From a practical point of view, the transmission factor $R_{0}$ is parametrized as $R_{0}=1+\rho / \sqrt{N}$. Then, Nåsell proposes to use the somewhat arbitrary value $\rho=3$ to determine the boundaries of the three regions.

On the other hand, in the case of the SIS model, the $R E$-distribution (3.5) reduces to

$$
P_{i}(j)=\frac{\frac{1}{j} \sum_{k=1}^{\min (i, j)}\left(\frac{R_{0}}{N}\right)^{j-k} \frac{(N-k) !}{(N-j) !}}{\sum_{j=1}^{N} \frac{1}{j} \sum_{k=1}^{\min (i, j)}\left(\frac{R_{0}}{N}\right)^{j-k} \frac{(N-k) !}{(N-j) !}}, 1 \leq i, j \leq N .
$$

At a first glance, we easily derive from (3.6) the epidemic behavior as far as the transmission factor increases but the population size remains constant. Then, we expect long absorption times while the mass probability is concentrated on the state $N$. This yields

$$
\begin{gathered}
\lim _{R_{0} \rightarrow \infty} E_{i}[T]=\infty, 1 \leq i \leq N, \\
\lim _{R_{0} \rightarrow \infty} P_{i}(j)=\delta_{j N}, 1 \leq i, j \leq N, \\
\lim _{R_{0} \rightarrow \infty} \sum_{j=1}^{N} j P_{i}(j)=N, 1 \leq i \leq N .
\end{gathered}
$$

We next summarize a set of stochastic ordering results which are useful to compare the quasi-stationary distribution, the $R E$-distribution and the birth and death approximations $p^{(0)}$ and $p^{(1)}$. For notational convenience, in the sequel we employ the bold notation $\mathbf{u}, \mathbf{P}_{i}$, for $1 \leq i \leq N, \mathbf{p}^{(0)}$ and $\mathbf{p}^{(1)}$ to denote the row vectors containing the probabilities of the corresponding distribution. The symbol $\leq_{s t}$ will denote the usual stochastic order relation with respect to the distribution function.

First, we notice that

$$
\mathbf{P}_{1}=\mathbf{p}^{(0)}
$$

as it was to be expected from the probabilistic interpretation of both distributions.

We now observe that

$$
\begin{gathered}
\mathbf{p}^{(0)} \leq_{s t} \mathbf{u} \leq_{s t} \mathbf{p}^{(1)} \\
\mathbf{P}_{1} \leq_{s t} \mathbf{u} \leq_{s t} \mathbf{P}_{N} \\
\mathbf{P}_{i} \leq_{s t} \mathbf{P}_{i^{\prime}}, 1 \leq i \leq i^{\prime} \leq N
\end{gathered}
$$

Relation $\mathbf{p}^{(0)} \leq_{s t} \mathbf{u}$ was proved by Cavender (1978) for the general birth and death process. For the SIS model, Kryscio and Lefèvre (1989) observed 
empirically that $\mathbf{p}^{(1)}$ is an upper bound of $\mathbf{u}$. This conjecture has been proved by Clancy and Pollett (2003) with the help of an auxiliary process that, whenever the system reaches the absorption, it automatically restarts in state $j$, for $1 \leq$ $j \leq N$, with probability $\nu_{j}$. In particular, by assigning unit mass to a fixed state $i$ (i.e., we take $\nu_{j}=\delta_{i j}$ ), we obtain formula (3.9); see Corollary 1 in Clancy and Pollett (2003). The result in (3.10) follows from Theorem 1 in that paper. It turns out that formulas (3.9) and (3.10) hold for the general birth and death process.

At this point, we also observe that the $R E$-distribution $\mathbf{P}_{i}$ is in fact equal to the stationary distribution of the auxiliary process with $\nu_{j}=\delta_{i j}$, for $1 \leq j \leq N$. In this way, we complete the circle arriving again at the probabilistic interpretation for $\mathbf{P}_{i}$ already given in Section 2, where we appealed to the relationship with the regenerative processes.

Formulas (3.8) and (3.9) show that $\mathbf{p}^{(1)}$ and $\mathbf{P}_{N}$ are upper bounds of the quasi-stationary distribution $\mathbf{u}$. Thus, it is appropriate to study the relation between them. To this end, for $N$ fixed, we find that

$$
\begin{gathered}
\lim _{R_{0} \rightarrow \infty} \frac{\sum_{j=1}^{l} p_{j}^{(1)}}{\sum_{j=1}^{l} P_{N}(j)}=\frac{l}{N} \leq 1,1 \leq l \leq N, \\
\lim _{R_{0} \rightarrow 0} \frac{\sum_{j=1}^{l} p_{j}^{(1)}}{\sum_{j=1}^{l} P_{N}(j)}=\frac{\sum_{j=1}^{N} \frac{1}{j}}{\sum_{j=1}^{l} \frac{1}{j}} \geq 1,1 \leq l \leq N,
\end{gathered}
$$

which amounts to the following relations:

$$
\begin{aligned}
& \mathbf{P}_{N} \leq s t \mathbf{p}^{(1)}, \text { for } N \text { fixed and } R_{0} \text { sufficiently large, } \\
& \mathbf{p}^{(1)} \leq_{s t} \mathbf{P}_{N}, \text { for } N \text { fixed and } R_{0} \text { sufficiently small. }
\end{aligned}
$$

From (3.9) we see that the extreme $R E$-distributions $\mathbf{P}_{1}$ and $\mathbf{P}_{N}$ are respectively lower and upper bounds of the quasi-stationary distribution $\mathbf{u}$. Moreover, formula (3.10) shows that the $R E$-distributions are stochastically monotone with respect to the initial state. Therefore, one may conjecture that either any appropriate choice of $i$ or the consideration of a mixture $\widetilde{\mathbf{P}}=\sum_{i=1}^{N} \omega_{i} \mathbf{P}_{i}$ would lead to some improvement, if one wishes to consider the $R E$-distribution as an approximation of the quasi-stationary distribution. In order to explore numerically this possibility, we next suggest two $R E$-approximations.

A first approximation, denoted by $\widehat{\mathbf{P}}$, approximates $\mathbf{u}$ by a single appropriate $R E$-distribution $\mathbf{P}_{i}$. The choice of the appropriate index $\widehat{i}$ depends on the three parameter regions described earlier. When $R_{0}$ is distinctly larger than 1 , we use the good approximation $\mathbf{p}^{(0)}$ to determine the starting state as $\widehat{i}=\left\langle\left\langle E\left[\mathbf{p}^{(0)}\right]\right\rangle\right\rangle$, where $\langle\langle x\rangle\rangle$ is the nearest integer function defined as the closest integer to $x$. In the parameter region where $R_{0}$ is distinctly smaller than 1 , we use $\mathbf{p}^{(1)}$ to get $\widehat{i}=\left\langle\left\langle E\left[\mathbf{p}^{(1)}\right]\right\rangle\right\rangle$. In the more involved transient region, we start from a rough but simple interpolation approximation $\overline{\mathbf{p}}=\frac{R_{0}}{1+R_{0}} \mathbf{p}^{(0)}+\frac{1}{1+R_{0}} \mathbf{p}^{(1)}$. Large values 
of the first weight give support to long extinction times, in agreement with the use of the approximation $\mathbf{p}^{(0)}$. Then, we take $\widehat{i}=\langle\langle E[\overline{\mathbf{p}}]\rangle\rangle$.

As a second approximation, we propose to employ a mixture of the $R E$ distributions, where the weights $\omega_{i}$ are given by the first $R E$-approximation $\widehat{\mathbf{P}}$. In this way, we construct the distribution $\widetilde{\mathbf{P}}$, which assigns probabilities $\widetilde{P}_{j}=\sum_{i=1}^{N} \widehat{P}_{i} P_{i}(j)$, for $1 \leq j \leq N$.

\begin{tabular}{|c|c|c|c|c|c|c|}
\hline$R_{0}$ & 0.5 & 0.9 & 1.0 & 1.3 & 1.5 & 2.0 \\
\hline$E[\mathbf{u}]$ & 1.9284 & 5.0459 & 7.0314 & 19.7010 & 30.8972 & 48.93045166 \\
\hline$\sigma(\mathbf{u})$ & 1.3152 & 3.9497 & 5.2233 & 8.9718 & 8.7257 & 7.23418980 \\
\hline$u_{1}$ & 0.5141 & 0.1732 & 0.1091 & 0.0092 & $4.2168 \times 10^{-4}$ & $7.8067 \times 10^{-9}$ \\
\hline $\mathbf{u}_{m}$ & 1 & 1 & 1 & 20 & 32 & 49 \\
\hline$E\left[\mathbf{p}^{(1)}\right]$ & 1.9444 & 5.5674 & 8.1900 & 23.2472 & 33.3362 & 50.00000001 \\
\hline$\sigma\left(\mathbf{p}^{(1)}\right)$ & 1.3312 & 4.2725 & 5.7378 & 8.5418 & 8.1589 & 7.07106775 \\
\hline$p_{1}^{(1)}$ & 0.5097 & 0.1501 & 0.0819 & 0.0022 & $4.4058 \times 10^{-5}$ & $3.2606 \times 10^{-10}$ \\
\hline $\mathbf{p}_{m}^{(1)}$ & 1 & 1 & 1 & 24 & 34 & 50 \\
\hline$E\left[\mathbf{P}_{1}\right]$ & 1.4251 & 2.8837 & 4.0986 & 17.9807 & 30.7926 & 48.93044999 \\
\hline$\sigma\left(\mathbf{P}_{1}\right)$ & 0.8602 & 2.7819 & 4.0950 & 9.7311 & 8.8501 & 7.23419422 \\
\hline$P_{1}(1)$ & 0.7264 & 0.4328 & 0.3356 & 0.0398 & 0.0013 & $1.5954 \times 10^{-8}$ \\
\hline $\mathbf{P}_{1}^{m}$ & 1 & 1 & 1 & 20 & 32 & 49 \\
\hline$E\left[\mathbf{P}_{N}\right]$ & 17.7858 & 16.2558 & 16.0297 & 20.8561 & 30.9337 & 48.93045195 \\
\hline$\sigma\left(\mathbf{P}_{N}\right)$ & 22.1172 & 18.3569 & 16.7672 & 10.4969 & 8.7764 & 7.23419022 \\
\hline$P_{N}(1)$ & 0.1292 & 0.0703 & 0.0538 & 0.0083 & $4.1967 \times 10^{-4}$ & $7.8065 \times 10^{-9}$ \\
\hline $\mathbf{P}_{N}^{m}$ & 1 & 1 & 1 & 21 & 32 & 49 \\
\hline$E[\mathbf{P}]$ & 1.8270 & 4.3226 & 6.4074 & 19.7563 & 30.8989 & 48.93045167 \\
\hline$\sigma(\widehat{\mathbf{P}})$ & 1.0522 & 3.2331 & 4.6079 & 8.8935 & 8.7229 & 7.23418979 \\
\hline$\widehat{P}_{1}$ & 0.4678 & 0.1736 & 0.1038 & 0.0087 & $4.2016 \times 10^{-4}$ & $7.8065 \times 10^{-9}$ \\
\hline$\widehat{\mathbf{P}}_{m}$ & 1 & 1 & 1 & 20 & 32 & 49 \\
\hline$E[\underset{\sim}{\widetilde{\mathbf{P}}}]$ & 1.7397 & 4.2893 & 6.2809 & 19.6495 & 30.8970 & 48.93045166 \\
\hline$\sigma(\widetilde{\mathbf{P}})$ & 1.1181 & 3.5146 & 4.9186 & 8.9897 & 8.7258 & 7.23418980 \\
\hline$\widetilde{P}_{1}$ & 0.5672 & 0.2232 & 0.1383 & 0.0096 & $4.2217 \times 10^{-4}$ & $7.8065 \times 10^{-9}$ \\
\hline$\widetilde{\mathbf{P}}_{m}$ & 1 & 1 & 1 & 20 & 32 & 49 \\
\hline$E_{1}[T]$ & 1.3765 & 2.3101 & 2.9789 & 25.1182 & 737.0896 & 62678430.2 \\
\hline$E_{N}[T]$ & 7.7396 & 14.2113 & 18.5840 & 120.4356 & 2382.7997 & 128096977.8 \\
\hline
\end{tabular}

Table 1. Comparing the characteristics of the $S I S$ model with $N=100$

Some numerical results are summarized in Tables 1 and 2. All codes have been done using Fortran. In Table 1 we compare six distributions, namely the quasi-stationary distribution $\mathbf{u}$, the birth and death approximation $\mathbf{p}^{(1)}$, the extreme $R E$-distributions $\mathbf{P}_{1}$ and $\mathbf{P}_{N}$, and the two $R E$-approximations $\widehat{\mathbf{P}}$ and $\widetilde{\mathbf{P}}$. From (3.7), we recall that $\mathbf{p}^{(0)}=\mathbf{P}_{1}$. We choose the population size as $N=100$ and normalize the recovery rate to be equal to one. Then, we take $R_{0}=0.5,0.9,1.0,1.3,1,5$ and 2.0. The choice here covers the three parameter 
regions. More concretely, we interpret that the cases $R_{0}=0.9$ and 1.0 belong to the transient region, while $R_{0}=1.3$ is somewhat in the boundary with the region where $R_{0}$ is distinctly larger than 1 . Each entry in the table gives, from top to bottom, the mean value, the standard deviation, the first probability and the mode, for the corresponding distribution. For example, in the case of $\mathbf{u}$ the notation of these characteristics is $E[\mathbf{u}], \sigma(\mathbf{u}), u_{1}$ and $\mathbf{u}_{m}$. Moreover, the last row gives the expected time to extinction from the states 1 and $N$. The numbers in bold indicate what is the closest distribution to the quasi-stationary one, with respect to the corresponding characteristic.

As a supplement to Table 1 , we notice that $R_{u}$ decreases from the value 0.95899 , for $R_{0}=0.5$, to the value $1.79 \times 10^{-8}$, corresponding to $R_{0}=2.0$. In fact, $R_{u}=0.99999$, for $R_{0}=10^{-9}$. Thus, we conclude that the quasi-stationary regime in the SIS model is always reached faster than the eventual absorption.

A summary of the observations inferred from Table 1 is given below.

- It is clear that $\mathbf{p}^{(1)}$ gives the best approximation in the region where $R_{0}$ is distinctly smaller than 1.

- Regarding the transient region, we remark the interest of an asymptotic approximation due to Nåsell (1999a), which is clearly better than $\mathbf{p}^{(0)}$ and $\mathbf{p}^{(1)}$. For the sake of simplicity, we have avoided the implementation of that approximation. Among the distributions under comparison here, we notice that the $R E$-approximations $\widehat{\mathbf{P}}$ and $\widetilde{\mathbf{P}}$ are satisfactorily close to $\mathbf{u}$ in the cases $R_{0}=$ 1.0 and 1.3. Thus, it seems reasonable to consider the use of $\widehat{\mathbf{P}}$ and $\widetilde{\mathbf{P}}$ in the interval $0 \leq \rho \leq 3$, specially when $N$ is moderate or small and there exist doubt about the applicability of asymptotic results.

- It is remarkable that all the distributions, except $\mathbf{p}^{(1)}$, are satisfactory approximations of $\mathbf{u}$ in the important practical region where $R_{0}$ is distinctly larger than 1. However, the best comparative indicators correspond to the distribution $\widetilde{\mathbf{P}}$. The differences among the distributions are more notorious as far as $\rho$ tends to 3 . In this case, it would be possible to use either $\widetilde{\mathbf{P}}$ or $\widehat{\mathbf{P}}$.

- Combining the stochastic ordering relationships (3.8)-(3.12) and the known asymptotic results (Nåsell 1996, 1999a), it is easy to conclude that the mean values of the six distributions under study are asymptotically equivalent to $N\left(R_{0}-1\right) / R_{0}$, in the region $R_{0}>1$. The numerical results in the table support this observation.

- Note that formula (7.3) in Nåsell (1996) gives the approximation $\widetilde{u}_{1}=$ $\sqrt{N}\left(R_{0}-1\right)^{2} \varphi\left(\beta_{1}\right) / R_{0}$, where $\varphi\left(\beta_{1}\right)=\frac{1}{\sqrt{2 \pi}} e^{-N \log R_{0}-\frac{R_{0}-1}{R_{0}}}$. When $R_{0}=1.3$, we have $\widetilde{u}_{1}=0.0169$, while $\widetilde{u}_{1}=8.1583 \times 10^{-9}$ for $R_{0}=2.0$. An explanation of the superiority of the $R E$-approximations over $\widetilde{u}_{1}$ is the following. From the approximation (8.4) for $E_{N}[T]$ in Nåsell (1996), we find that $P_{N}(1)$ can be approximated by $\widetilde{P}_{N}(1)=\sqrt{N} R_{0}^{N-1}\left(R_{0}-1\right)^{2} \varphi\left(\beta_{1}\right) /\left(R_{0}^{N}-1\right)$. Now we observe that $\widetilde{u}_{1}<\widetilde{P}_{N}(1)$ but this disagrees with formula (3.9), which implies the exact relation $P_{N}(1) \leq u_{1}$. The interest in the first probability $u_{1}$ comes from its 
relation with the expected time to extinction when the initial distribution is $\mathbf{u}$. Denoting the latter as $E\left[T_{u}\right]$, we recall that both quantities are related through the formula $E\left[T_{u}\right]=\left(\gamma u_{1}\right)^{-1}$.

In Table 2, we employ the maximum pointwise distance to compare the distributions under consideration versus the quasi-stationary distribution. For example, in the case of $\mathbf{p}^{(1)}$, the distance is defined by $\left|\mathbf{p}^{(1)}-\mathbf{u}\right|=\max _{1 \leq j \leq N}\left|p_{j}^{(1)}-u_{j}\right|$. The entry marked in bold is again associated with the distribution providing the minimum distance and, consequently, the best global approximation.

\begin{tabular}{|c|c|c|c|c|c|c|}
\hline$R_{0}$ & 0.5 & 0.9 & 1.0 & 1.3 & 1.5 & 2.0 \\
\hline $\mathbf{p}^{(1)}-\mathbf{u}$ & 0.004405 & 0.050911 & 0.087469 & 0.161472 & 0.111504 & 0.058900 \\
\hline$\left|\mathbf{P}_{1}-\mathbf{u}\right|$ & 0.212319 & 0.303681 & 0.302958 & 0.078784 & 0.003570 & $4.2672 \times 10^{-8}$ \\
\hline$\left|\mathbf{P}_{N}-\mathbf{u}\right|$ & 0.588294 & 0.375155 & 0.288864 & 0.032600 & 0.001074 & $1.0384 \times 10^{-8}$ \\
\hline$\widehat{\mathbf{P}}-\mathbf{u}$ & 0.051347 & 0.091901 & 0.063566 & 0.005462 & $1.4852 \times 10^{-4}$ & $9.2142 \times 10^{-10}$ \\
\hline$\widetilde{\mathbf{P}}-\mathbf{u}$ & 0.055082 & 0.086271 & 0.064538 & 0.002328 & $5.5914 \times 10^{-6}$ & $9.0205 \times 10^{-16}$ \\
\hline
\end{tabular}

Table 2. Distributions distances with respect to $\mathbf{u}$

In the light of the table, we see that the best approximation starts being $\mathbf{p}^{(1)}$, in the region $R_{0}<1$, but passes by $\widehat{\mathbf{P}}$, in the transient region, and finally becomes $\widetilde{\mathbf{P}}$, in the region $R_{0}>1$. It should be pointed out the significant improvement obtained in the region $R_{0}>1$ when we use $\widetilde{\mathbf{P}}$.

\subsubsection{A linear growth model}

As an example of the infinite case $N=\infty$, we next consider the linear growth model with transition rates

$$
\begin{aligned}
& \lambda_{i}=\lambda i, \quad i \geq 0, \\
& \mu_{i}=\mu i, \quad i \geq 1 .
\end{aligned}
$$

Linear transition rates arise often in combination with catastrophes, see for instance Lee (2000). Moreover, the linear growth model is one of the few cases where both the quasi-stationary distribution and the $R E$-distribution can be determined explicitly (Nåsell, 2001). This fact facilitates a rapid comparison between both distributions.

We assume that $R_{0}=\lambda / \mu<1$. Then, the quasi-stationary distribution follows the geometric law:

$$
u_{j}=\left(1-R_{0}\right) R_{0}^{j-1}, j \geq 1 .
$$

On the other hand, formula (3.5) for the $R E$-distribution yields 


$$
P_{i}(j)= \begin{cases}\frac{\left(1-R_{0}^{j}\right) j^{-1}}{\left(1-\delta_{1 i}\right){ }_{j=1}^{i-1} \frac{1-R_{0}^{j-i}}{j}-\frac{1-R_{0}^{i}}{R_{0}^{i}} \ln \left(1-R_{0}\right)}, & \text { if } 1 \leq j<i, \\ \frac{R_{0}^{j-i}\left(1-R_{0}^{i}\right) j^{-1}}{\left(1-\delta_{1 i}\right)}{ }_{j=1}^{i-1} \frac{1-R_{0}^{j-i}}{j}-\frac{1-R_{0}^{i}}{R_{0}^{i}} \ln \left(1-R_{0}\right) & \text { if } 1 \leq i \leq j .\end{cases}
$$

It is easy to show that both distributions have a unique mode at the point $\mathbf{u}_{m}=\mathbf{P}_{i}^{m}=1$, for $i \geq 1$, whereas the expected values are given by

$$
\begin{gathered}
\sum_{j=1}^{\infty} j u_{j}=\frac{1}{1-R_{0}}, \\
\sum_{j=1}^{\infty} j P_{i}(j)=i\left(\left(1-\delta_{1 i}\right) \sum_{j=1}^{i-1} \frac{1-R_{0}^{j-i}}{j}-\frac{1-R_{0}^{i}}{R_{0}^{i}} \ln \left(1-R_{0}\right)\right), i \geq 1 .
\end{gathered}
$$

We are interested in the limiting behavior as $R_{0} \rightarrow 1$. Then, we obtain the following limiting results:

$$
\begin{gathered}
\lim _{R_{0} \rightarrow 1} E_{i}[T]=\infty, i \geq 1, \\
\lim _{R_{0} \rightarrow 1} \sum_{j=1}^{\infty} j u_{j}=\infty, \\
\lim _{R_{0} \rightarrow 1} \sum_{j=1}^{\infty} j P_{i}(j)=\infty, i \geq 1, \\
\lim _{R_{0} \rightarrow 1} \frac{\sum_{j=1}^{\infty} j P_{i}(j)}{\sum_{j=1}^{\infty} j P_{i^{\prime}}(j)}=1,1 \leq i, i^{\prime} \leq N, \\
\lim _{R_{0} \rightarrow 1} \frac{\sum_{j=1}^{\infty} j P_{i}(j)}{\sum_{j=1}^{\infty} j u_{j}}=0, i \geq 1 .
\end{gathered}
$$

Formula (3.13) says that the expected absorption time becomes arbitrarily long, as far as $R_{0} \rightarrow 1$, so the comparison is meaningful. In agreement with the intuition, formulas (3.14) and (3.15) show that the mean value of the two distributions tends to infinity. From (3.15), we also notice that the influence of the initial state $i$ vanishes when the time to absorption is long. Formula (3.16) says that the mean values of the $R E$-distribution obtained for two different initial states are asymptotically equivalent, when $R_{0} \rightarrow 1$. Finally, the limiting ratio in (3.17) illustrates the important fact that the mean value of the quasistationary distribution converges to infinity faster than the mean value of the 
$R E$-distribution. In this average sense, a similar limiting behavior of both distributions cannot be concluded.

\subsection{SIR epidemic models}

The $S I R$ formalism categorizes individuals within a closed population as susceptible, infective and removed individuals. The main difference between $S I S$ and $S I R$ models is that, in the $S I R$ model, individuals recover and develop permanent immunity. We are concerned with the classical $S I R$ model and with a finite $S I R$ model with demography. The classical $S I R$ model refers to the standard formulation as it can be found in many textbooks (Andersson and Britton, 2000; Allen, 2003; Bailey, 1990; Daley and Gani, 1999). The introduction of demographic forces in the epidemiological formalism was already proposed by Bartlett (1956). Slight variants of Bartlett's model lead to what is known as the $S I R$ model with demography (van Herwaarden and Grasman, 1995; Nåsell, 1999b). These models and their generalizations (see e.g. Nåsell, 2002) are based on Markov chains with infinite state space. In Subsection 3.2 .2 , we introduce a finite $S I R$ model with demography. The finitess of the state space makes the model enough tractable, which is needed to deal with our objective of comparing the quasi-stationary distribution and the ratio of expectations distribution.

The existence of a finite state space, which can be partitioned into two classes of absorbing and transient states, is a common feature for the two SIR models under study, and even for the classical SIS model. However, the quasistationary behavior of these models is completely different due to the different dynamics of the transitions among the transient states.

\subsubsection{The classical SIR model}

Let $\{(X(t), Y(t)) ; t \geq 0\}$ be the bidimensional continuous-time Markov chain describing the $S I R$ epidemic model. At time $t$, the population consists of $X(t)$ infectives, $Y(t)$ susceptibles and $Z(t)=N-X(t)-Y(t)$ immune individuals, where $N$ is the constant population size. The initial condition is $(X(0), Y(0))=$ $(m, n)$. When in state $(i, j)$, for $i \geq 1$, the population state moves either to $(i+1, j-1)$ at rate $\lambda_{i j}\left(\lambda_{i 0}=0\right)$ due to an infection, or to $(i-1, j)$ at rate $\mu_{i}\left(\mu_{0}=0\right)$ due to the removal of an infective. The state space of the $S I R$ epidemic model is $S=\{(i, j) ; 0 \leq i \leq m+n, 0 \leq j \leq \min \{n, m+n-i\}\}$. We notice that states $(0, j)$, for $0 \leq j \leq n$, are absorbing states, so it is reasonable to assume that $m \geq 1$. Fig. 2 illustrates the transitions among the states for the case $(m, n)=(3,3)$. 


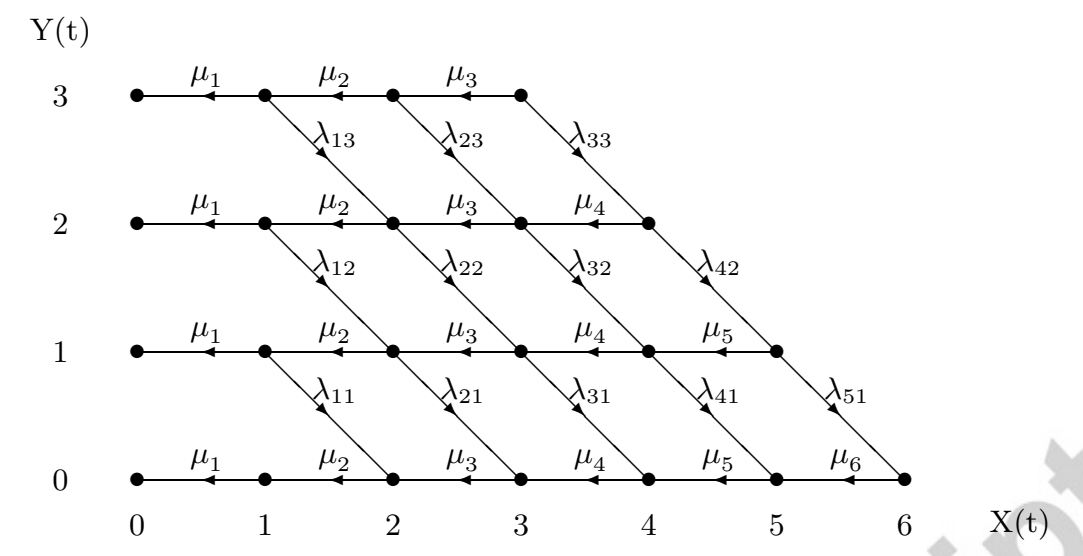

Fig. 2. States and transitions of the SIR epidemic model

A typical choice for the transition rates is (Allen, 2003)

$$
\begin{gathered}
\lambda_{i j}=\frac{\beta}{N} i j, \quad(i, j) \in S, \\
\mu_{i}=\gamma i, 0 \leq i \leq m+n,
\end{gathered}
$$

where $\beta$ and $\gamma$ denote the contact and the recovery rates.

From the results summarized in Section 2 it follows easily that the quasistationary distribution only assigns positive probabilities to the states $(i, 0)$ with $1 \leq i \leq i^{*}$, where $i^{*}$ is the minimum $i$, for $1 \leq i \leq m+n$, such that $\mu_{i}=\min _{1 \leq k \leq m+n} \mu_{k}$. In particular, if $\mu_{i}=\gamma i$, for $1 \leq i \leq m+n$, then $i^{*}=1$ and the quasi-stationary distribution has the trivial form

$$
u_{(i, j)}=\delta_{(1,0)(i, j)}, 0 \leq j \leq n, 1 \leq i \leq m+n-j,
$$

that is, all the probability mass is concentrated at the state $(1,0)$.

On the other hand, the $R E$-distribution of the $S I R$ epidemic model has the form

$$
P_{(m, n)}(i, j)=\frac{E_{(m, n)}\left[T_{(i, j)}\right]}{E_{(m, n)}[T]}, 0 \leq j \leq n, 1 \leq i \leq m+n-j .
$$

Define $A_{i j}$ to be the probability of reaching the state $(i, j) \in S$ starting from $(m, n)$ before the extinction occurs. Considering that each state $(i, j)$ can be visited at most one time, we write formula (3.18) as follows:

$$
P_{(m, n)}(i, j)=\frac{\frac{A_{i j}}{\lambda_{i j}+\mu_{i}}}{\sum_{j=0}^{n} \sum_{i=1}^{m+n-j} \frac{A_{i j}}{\lambda_{i j}+\mu_{i}}}, 0 \leq j \leq n, 1 \leq i \leq m+n-j
$$


Next we summarize the recursive scheme for the computation of the probabilities $A_{i j}$ (Neuts and Li, 1996).

Step 1. Set $A_{i j}=\delta_{(i, j)(m, n)}$.

Step 2. For $i=m-1, m-2, \ldots, 0$ calculate

$$
A_{i n}=A_{i+1, n} \frac{\mu_{i+1}}{\lambda_{i+1, n}+\mu_{i+1}} .
$$

Step 3. For $k=1$, calculate

$$
\begin{aligned}
& \text { 3.a. } \\
& \qquad \begin{array}{l}
A_{m+k, n-k}=A_{m+k-1, n-k+1} \frac{\lambda_{m+k-1, n-k+1}}{\lambda_{m+k-1, n-k+1}+\mu_{m+k-1}} \\
\text { 3.b. For } i=m+k-1, m+k-2, \ldots, 2 \text { compute } \\
A_{i, n-k}=A_{i+1, n-k} \frac{\mu_{i+1}}{\lambda_{i+1, n-k}+\mu_{i+1}}+A_{i-1, n-k+1} \frac{\lambda_{i-1, n-k+1}}{\lambda_{i-1, n-k+1}+\mu_{i-1}} .
\end{array}
\end{aligned}
$$

3.c For $i=0,1$ calculate

$$
A_{i, n-k}=A_{i+1, n-k} \frac{\mu_{i+1}}{\lambda_{i+1, n-k}+\mu_{i+1}} .
$$

Step 4. Set $k=k+1$. If $k \leq n$ go to Step 3.a.

We notice that the above recursive scheme also provides a method for computing the final size of the epidemic. In fact, $A_{0 j}$, for $0 \leq j \leq n$, gives the probability that there are $j$ susceptible individuals at the extinction time. Thus, the final size of the epidemic is $N-j$.

At this point, we would like to note that the $R E$-distribution gives positive probability to all transient states $S_{T}=S-\{(0, j) ; 0 \leq j \leq n\}$. In contrast, the typical choice of the $S I R$ gives all the quasi-stationary probability mass to $(1,0)$. As in the example for the simple death process showed in Section 2, the explanation is related to the fact that $S_{T}$ is reducible, but also to the fact that state $(1,0)$ has the lowest exit rate (i.e., $\min _{(i, j) \in S_{T}}\left(\lambda_{i j}+\mu_{i}\right)=\lambda_{10}+\mu_{1}=$ $\gamma)$. Moreover, once a state has been visited, the system leaves it forever. In this sense, the $S I R$ formalism is more appropriate to model acute infections including chickenpox, measles, mumps and rubella (Keeling and Rohani (2008)), where in a relatively rapid period the extinction time is reached. The ratio $R_{u}$ gives support to the same idea. It is easy to prove that the eigenvalues of $Q_{S_{T}}$ in the $S I R$ model are given by $-\left(\lambda_{i j}+\mu_{i}\right)$, for $0 \leq j \leq n$ and $1 \leq i \leq m+n-j$. Then, we readily find that $R_{u} \geq 1$. More concretely, we have

$$
R_{u}= \begin{cases}1, & \text { if } \gamma \leq \beta / N \\ \frac{2 \gamma}{\beta / N+\gamma}, & \text { if } \gamma>\beta / N\end{cases}
$$


In the light of the above comments, it should be erroneous to conclude that the concentration of the mass in a single point is a drawback of the quasistationarity. The correct interpretation is that it is not meaningful to study the quasi-stationary distribution in this case, because probably the time to absorption is not long enough. In contrast, the $R E$-distribution can be used despite how long the absorption time is, so it provides a natural approach to measure the behavior of the $S I R$ model before absorption. Fortunately, we have more than one option to cover our basic objective.

\subsubsection{A finite SIR model with demography}

In the SIR model with demography (Nåsell, 1999b), the population is subject to immigration and deaths. It is usual to assume a constant birth rate $\mu N$ and a linear death rate $\mu j$, proportional to the number $j$ of susceptibles. Note that $N$ is not the total population size. In fact, the population size varies along the time, but $N$ can be viewed as the mean equilibrium population size when the infection has been eradicated. The state space of the Markov chain modelling the $S I R$ model with demography is $S=\mathbb{N} \times \mathbb{N}$ and the states $\{(0, j) ; j \geq 0\}$ form the absorbing set $S_{A}$. In this context, the quasi-stationary distribution cannot be determined neither explicit nor recursively. The study of the time to extinction is also intricate, even to prove almost sure extinction is an involved problem. The existing work (Nåsell, 1999b, 2002) is mainly concerned with the determination of asymptotic approximations of the quasi-stationary distribution and the time to extinction, when the ratio $R_{0}$ is distinctly larger than 1 . We next consider alternative demographic rates leading to a Markov chain with finite state space $S=\{(i, j) ; 0 \leq i+j \leq N\}$. With this state space, the comparative study of the quasi-stationary and the ratio of expectations distributions can be numerically implemented.

We consider a bidimensional process $\{(X(t), Y(t)) ; t \geq 0\}$, where $X(t)$ denotes the number of infected individuals, while $Y(t)$ represents the number of susceptibles at time $t$. When in state $(i, j)$, the dynamics of the system is defined by the following four transitions. Due to an infection the population size moves to $(i+1, j-1)$ at rate $\lambda_{i j}=\beta i j / N$, while the removal of an infective causes a transition to $(i-1, j)$ at rate $\mu_{i}=(\gamma+\mu+\theta) i$. Here $\beta$ and $\gamma$ represent the contact and the recovery rate, as in the classical SIR model. The rates $\mu$ and $\theta$ correspond to natural death and death due to the infection, respectively. Due to the demographic forces the system moves either to state $(i, j+1)$ at rate $\alpha_{i j}=\left(\frac{\lambda}{N}(i+j)+\xi\right)(N-i-j)$, when a birth takes places, or to $(i, j-1)$ at rate $\delta_{j}=\mu j$, due to the death of a susceptible. The rate $\alpha_{i j}$ can be decomposed into two contributions. The first term $\frac{\lambda}{N}(i+j)(N-i-j)$ shows that the internal births follow a logistic growth. With this rate, the population cannot growth beyond the carrying capacity $N$. On the other hand, the term $\xi(N-i-j)$ gives the rate of the external (immigration) births. We assume that the arrival of immigrants decreases as far as the population tends to its ideal carrying capacity. The transition among states are represented in Figure 3, for the simple case $N=3$. 


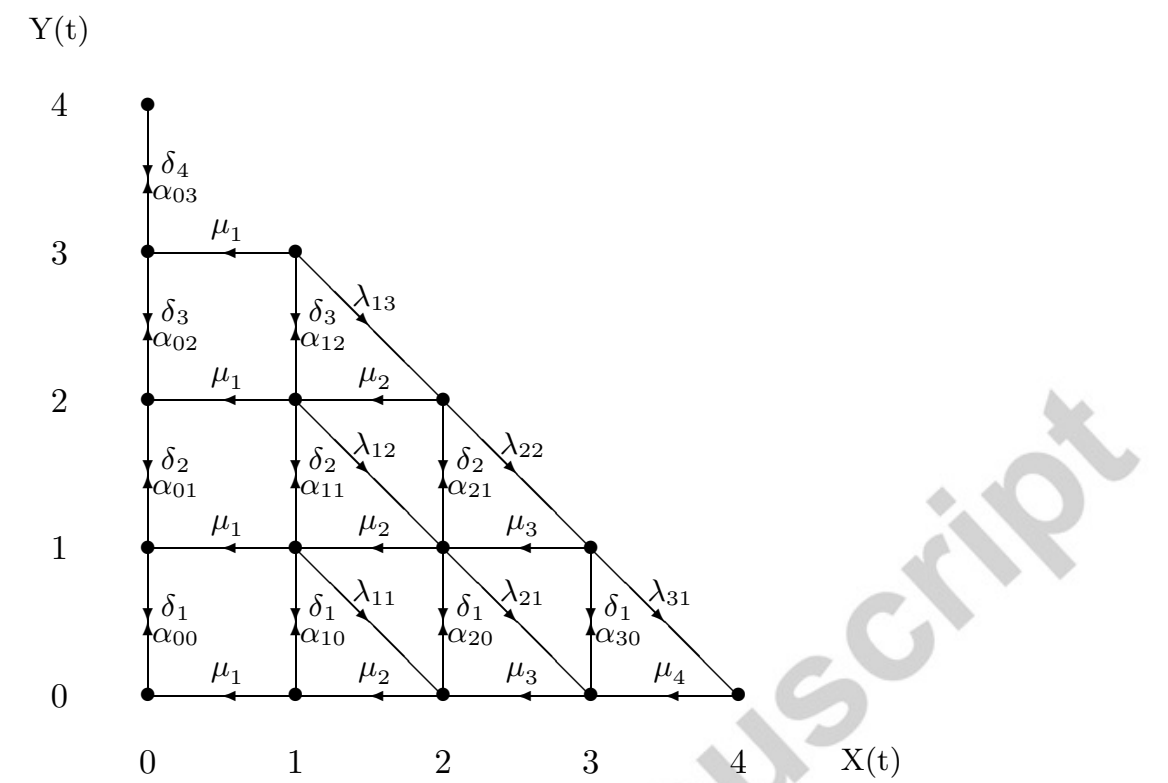

Fig. 3. States and transitions of the SIR model with demography

As a partially related work, we mention the papers by Swift (2001) and Allen and Burgin (2000). The former deals with a model operating in the presence of logistic growth with immigration. In the latter, an SIR model with finite triangular state space is considered. In that paper, each death is accompanied by a birth so that the population size remains constant.

In Table 3, we illustrate the main characteristics of the quasi-stationary probabilities $u_{i j}$, for $(i, j) \in S_{T}=\{(i, j) ; 1 \leq i+j \leq N\}$, which can be computed from the fact that the quasi-stationary distribution $\mathbf{u}$ is the left eigenvector of $Q_{S_{T}}$ with maximal real part. The finite $S I R$ model with demography has seven system parameters: $N, \beta, \gamma, \mu, \theta, \lambda$ and $\xi$. In our numerical experiment, we fix $N=100, \gamma=499 / 500, \mu=1 / 500, \theta=0$ and $\xi=0.1$. The choice of $\gamma$ and $\mu$ normalizes $\gamma+\mu$ as 1 , and reflects that the expected life is much longer than the expected time to recovery. The internal birth rate $\lambda$ is chosen to be multiple of $\xi$; that is, we consider $\lambda=0.1,0.5,1.0,5.0$ and 10.0. Finally, the transmission factor $R_{0}=\beta /(\gamma+\mu)=\beta$ takes values $R_{0}=0.5,1.0,1.25,1.5$ and 2.0. 


\begin{tabular}{|c|c|c|c|c|c|}
\hline$E\left[\mathbf{u}_{i .}\right]$ & & & & & \\
$E\left[\mathbf{u}_{. j}\right]$ & $R_{0}=0.5$ & $R_{0}=1.0$ & $R_{0}=1.25$ & $R_{0}=1.5$ & $R_{0}=2.0$ \\
$\sigma\left(\mathbf{u}_{i .}\right)$ & & & & & \\
$\mathbf{u}_{i j}^{m}$ & & & & & \\
\hline & 1.782729 & 3.358695 & 4.310522 & 5.197538 & 6.548307 \\
$\lambda=0.1$ & 89.880561 & 75.847244 & 68.015593 & 60.676247 & 48.776272 \\
& 1.157914 & 2.531369 & 3.200962 & 3.745675 & 4.447316 \\
& $(1,92)$ & $(1,82)$ & $(1,76)$ & $(1,69)$ & $(2,55)$ \\
\hline & 1.868709 & 4.580918 & 7.014187 & 9.664507 & 13.639154 \\
$\lambda=0.5$ & 95.195758 & 85.992502 & 78.088749 & 69.072884 & 53.392711 \\
& 1.247307 & 3.416681 & 4.718968 & 5.681166 & 6.534392 \\
& $(1,97)$ & $(1,94)$ & $(1,92)$ & $(7,74)$ & $(14,50)$ \\
\hline & 1.893701 & 5.279383 & 9.141594 & 13.925720 & 21.204501 \\
$\lambda=1.0$ & 96.527713 & 89.140401 & 80.996214 & 70.534363 & 52.768775 \\
& 1.274471 & 3.913796 & 5.704721 & 6.792541 & 7.085032 \\
& $(1,98)$ & $(1,97)$ & $(1,97)$ & $(13,72)$ & $(22,51)$ \\
\hline & 1.920373 & 6.495080 & 14.149327 & 25.043827 & 39.970584 \\
$\lambda=5.0$ & 97.753647 & 92.160278 & 82.903763 & 69.670016 & 51.334353 \\
& 1.305275 & 4.813970 & 7.704323 & 8.319430 & 7.237475 \\
& $(1,99)$ & $(1,99)$ & $(1,99)$ & $(25,70)$ & $(40,52)$ \\
\hline & 1.924342 & 6.744306 & 15.386401 & 27.732683 & 44.159848 \\
$\lambda=10.0$ & 97.912692 & 92.561982 & 83.026944 & 69.391751 & 51.195365 \\
& 1.310106 & 5.003532 & 8.137786 & 8.526345 & 7.238408 \\
& $(1,99)$ & $(1,99)$ & $(1,99)$ & $(27,71)$ & $(45,51)$ \\
\hline
\end{tabular}

Table 3. Characteristics of the quasi-stationary distribution

For each pair $\left(R_{0}, \lambda\right)$, we compute the marginal expected values of the number of infectives, $E\left[\mathbf{u}_{i .}\right]$, and the number of susceptibles, $E\left[\mathbf{u}_{. j}\right]$, the standard deviation of the number of infectives, $\sigma\left(\mathbf{u}_{i}\right.$.), and the mode $\mathbf{u}_{i j}^{m}$ (i.e., the bidimensional pair $(i, j)$ with highest quasi-stationary mass). An examination of the table reveals that $E\left[\mathbf{u}_{i}.\right]$ is an increasing function of $R_{0}$ and $\lambda$. The mean value $E\left[\mathbf{u}_{. j}\right]$ decreases as a function of $R_{0}$ and it has a mode as a function of $\lambda$, in the region $R_{0}>1$. The standard deviation $\sigma\left(\mathbf{u}_{i}\right)$ and the mode $\mathbf{u}_{i j}^{m}$ exhibit higher magnitudes in the southeastern corner of the table.

In Table 4 we are concerned with the $\widehat{\mathbf{P}}$ distribution obtained by starting the $R E$-distribution $P_{(m, n)}(i, j)$ at the point $(\widehat{m}, \widehat{n})$, such that $\widehat{m}=\left\langle\left\langle E\left[\mathbf{u}_{i .}\right]\right\rangle\right\rangle$ and $\widehat{n}=\left\langle\left\langle E\left[\mathbf{u}_{. j}\right]\right\rangle\right\rangle$. The $\widehat{\mathbf{P}}$ distribution can be computed by solving numerically the systems of linear equations that govern the expectations $E_{(m, n)}\left[T_{(i, j)}\right]$ and $E_{(m, n)}[T]$. The block tridiagonal structure of the infinitesimal generator might be exploited to this end. 


\begin{tabular}{|c|c|c|c|c|c|}
\hline$E\left[\widehat{\mathbf{P}}_{i .}\right]$ & & & & & \\
$E\left[\widehat{\mathbf{P}}_{. j}\right]$ & $R_{0}=0.5$ & $R_{0}=1.0$ & $R_{0}=1.25$ & $R_{0}=1.5$ & $R_{0}=2.0$ \\
$\sigma\left(\widehat{\mathbf{P}}_{i .}\right)$ & & & & & \\
$\widehat{\mathbf{P}}_{i j}^{m}$ & & & & & \\
\hline & 1.761919 & 3.069476 & 4.085833 & 5.080829 & 6.600214 \\
$\lambda=0.1$ & 90.365396 & 77.185894 & 69.161475 & 61.606620 & 48.957257 \\
& 0.972266 & 2.149048 & 2.854446 & 3.446865 & 4.186577 \\
& $(2,90)$ & $(3,76)$ & $(4,68)$ & $(5.61)$ & $(7,49)$ \\
\hline \multirow{5}{*}{$\lambda=0.5$} & 1.802639 & 4.563704 & 7.013047 & 9.768168 & 13.674764 \\
& 95.409072 & 86.440183 & 78.341271 & 68.935081 & 53.309221 \\
& 1.021293 & 3.140335 & 4.523125 & 5.568556 & 6.477212 \\
& $(2,95)$ & $(5,86)$ & $(7,78)$ & $(10,69)$ & $(14,53)$ \\
\hline & 1.815385 & 5.001877 & 9.140858 & 13.982033 & 21.206802 \\
$\lambda=1.0$ & 96.899195 & 89.731851 & 81.066132 & 70.447300 & 52.764619 \\
& 1.036569 & 3.514131 & 5.529311 & 6.734425 & 7.080823 \\
& $(2,97)$ & $(1,97)$ & $(9,81)$ & $(14,71)$ & $(22,51)$ \\
\hline & 1.824432 & 6.076221 & 14.174662 & 25.049965 & 39.970584 \\
$\lambda=5.0$ & 97.895901 & 92.652233 & 82.874674 & 69.662724 & 51.334353 \\
& 1.048449 & 4.294910 & 7.556473 & 8.310091 & 7.237474 \\
& $(1,99)$ & $(1,99)$ & $(1,99)$ & $(25,70)$ & $(40,52)$ \\
\hline & 1.825670 & 6.547522 & 15.398180 & 27.736629 & 44.159848 \\
$\lambda=10.0$ & 98.026322 & 92.785416 & 83.013491 & 69.387390 & 51.195365 \\
& 1.050229 & 4.542308 & 7.996709 & 8.520852 & 7.238408 \\
& $(1,99)$ & $(1,99)$ & $(13,86)$ & $(27,71)$ & $(45,51)$ \\
\hline
\end{tabular}

Table 4. Characteristics of the $\widehat{\mathbf{P}}$ distribution

From our numerical experiments, we now observe that $R_{u}<1$ for all choices of the pair $\left(R_{0}, \lambda\right)$ in the tables, except for the case $\left(R_{0}, \lambda\right)=(0.5,0.1)$, where $R_{u}=1.41443$. As a result, the comparison between $\widehat{\mathbf{P}}$ and $\mathbf{u}$ is meaningful in a broad domain of the considered parameters.

By comparing the entries in Tables 3 and 4, we may conclude that $\widehat{\mathbf{P}}$ provides an acceptable approximation of $\mathbf{u}$ in the whole range of the pair $\left(R_{0}, \lambda\right)$. The quality of the approximation is improved when $R_{0}$ and $\lambda$ simultaneously increase and, consequently, the time to extinction becomes longer. In fact, both distributions present identical values in the case $\left(R_{0}, \lambda\right)=(2.0,10.0)$. Numerical experiments, not reported here, show that the characteristics of the $\widehat{\mathbf{P}}$ distribution are almost insensitive with respect to the initial state, as far as $R_{0}$ and $\lambda$ increase.

\section{Concluding comments}

Our aim in this paper is to provide a comparative analysis of the quasistationary and the ratio of expectation distributions. The motivation for this study comes from the need of understanding the behavior of a biological stochastic system before absorption. For many applications, it is certain that the ab- 
sorption occurs but this may take a very long time. Then, the quasi-stationary distribution gives an excellent measure of the long-term behavior of the system. Due to the non-linear structure of the quasi-stationary equations, it is usually impossible to obtain explicit expressions for the quasi-stationary distribution. However, there exists a number of helpful results including recursive methods, approximations and asymptotic analysis. The $R E$-distribution gives an alternative to measure the system dynamics before absorption, despite of how long the absorption time is. Since the $R E$-distribution is governed by linear equations, it can typically be evaluated more simply. The main problem of the $R E$-distribution concerns the practical difficulties for managing information about the initial distribution.

Several scenarios (classical SIS model, a linear growth model, classical SIR model, a finite $S I R$ model with demography) are considered along the paper to investigate a two-fold objective: $i$ ) to consider the quasi-stationarity and the ratio of expectations as two conceptually different approaches for measuring the behavior of a biological system before reaching the absorbing states, and $i i$ ) to evaluate the possibility of using the $R E$-distribution as an approximation to the quasi-stationary distribution provided that the quasi-stationary regime has already been reached.

The study can be continued in several directions. For example, it would be interesting to get asymptotic expansions for the expectations $E_{i}\left[T_{j}\right]$. If we allow absorption in one transition we arrive to the consideration of biological models with killing and catastrophes (Coolen-Schrijner and van Doorn, 2006; Artalejo et al., 2007). In the present paper, the applications of the $R E$-distribution were oriented to epidemic models but a forthcoming study might explore other important stochastic biological models including competition and predation (Allen, 2003).

We also notice that the approach can be easily extended to the discretetime case. Similar arguments to those given in Section 2 lead to a system of equations for the number of visits to state $j$ before absorption. In fact, the discrete counterpart follows by replacing the infinitesimal rates $q_{i j}$ by the onestep transition probabilities. Then, the analogue to equation (2.8) takes the form

$$
\left(I-P_{S_{T}}\right) \mathbf{m}_{j}=\mathbf{e}_{j},
$$

where $P_{S_{T}}$ denotes the one-step transition probability sub-matrix corresponding to the transient states.

\section{Acknowledgements}

This research was supported by the Spanish Government (Department of Education and Innovation) and the European Commission through project MTM 2008-01121. The authors would like to thank the referees for their helpful com- 
ments and suggestions which greatly improved the paper.

\section{References}

Allen, L.J.S., Burgin, A.M., 2000. Comparison of deterministic and stochastic $S I S$ and SIR models in discrete time. Math. Biosci. 163, 1-33.

Allen, L.J.S., 2003. An Introduction to Stochastic Processes with Applications to Biology. Prentice-Hall, Upper Saddle River, New Jersey.

Allen, L.J.S., 2008. An Introduction to Stochastic Epidemic Models. Lecture Notes in Mathematics, vol. 1945, Springer, Berlin, pp. 81-130.

Andersson, H., Britton, T., 2000. Stochastic Epidemic Models and Their Statistical Analysis. Lecture Notes in Statistics, vol. 151, Springer, New York.

Artalejo, J.R., Economou, A., Lopez-Herrero, M.J., 2007. Evaluating growth measures in an immigration process subject to binomial and geometric catastrophes. Math. Biosci. Eng. 4, 573-594.

Bailey, N.T.J., 1990. The Elements of Stochastic Processes with Applications to the Natural Sciences. John Wiley \& Sons, New York.

Bartlett, M.S., 1956. Deterministic and stochastic models for recurrent epidemics. In: Proc. Third Berkeley Symp. Math. Statist. \& Prob. 4, Univ. California Press. Berkeley and Los Angeles 4, 81-109.

Breyer, L.A., Hart, A.G., 2000. Approximations of quasi-stationary distributions for Markov chains. Math. Comput. Model. 31, 69-79.

Casagrandi, R., Gatto, M., 2006. The intermediate dispersal principle in spatially explicit metapopulations. J. Theor. Biol. 239, 22-32.

Cavender, J.A., 1978. Quasi-stationary distributions of birth-and-death processes. Adv. Appl. Prob. 10, 570-586.

Clancy, D., Pollett, P.K., 2003. A note on quasi-stationary distributions of birth-death processes and the SIS logistic epidemic. J. Appl. Prob. 40, 821825 .

Clancy, D., 2005. A stochastic SIS infection model incorporating indirect transmission. J. Appl. Prob. 42, 726-737.

Clancy, D., O'Neill, P.D., 2008. Bayesian estimation of the basic reproduction number in stochastic epidemic models. Bayesian Anal. 3, 737-758.

Cohen, A.M., 2007. Numerical Methods for Laplace Transform Inversion. Springer, New York.

Coolen-Schrijner, P., van Doorn, E., 2006. Quasi-stationary distributions for a class of discrete-time Markov chains. Methodol. Comput. Appl. Probab. 8, 449-465.

Daley, D.J., Gani, J., 1999. Epidemic Modelling: An Introduction. Cambridge Studies in Mathematical Biology 15. Cambridge University Press, Cambridge.

Darroch, J.N., Seneta, E., 1965. On quasi-stationary distributions in absorbing discrete-time finite Markov chains. J. Appl. Prob. 2, 88-100.

Darroch, J.N., Seneta, E., 1967. On quasi-stationary distributions in absorbing 
continuous-time finite Markov chains. J. Appl. Prob. 4, 192-196.

Ewens, W.J., 1963. The diffusion equation and a pseudo-distribution in genetics. J. R. Statist. Soc. B 25, 405-412.

Fouchet, D., Guitton, J.S., Marchandeau, S., Pontier, D., 2008. Impact of myxomatosis in relation to local persistence in wild rabbit populations: The role of waning immunity and the reproductive period. J. Theor. Biol. 250, 593-605.

Keeling, M.J., Rohani, P., 2008. Modeling Infectious Diseases in Humans and Animals. Princeton University Press, Princeton.

Kryscio, R.J., Lefèvre, C., 1989. On the extinction of the $S-I-S$ stochastic logistic epidemic. J. Appl. Prob. 27, 685-694.

Kulkarni, V.G., 1995. Modeling and Analysis of Stochastic Systems. Chapman \& Hall, London.

Lee, C., 2000. The density of the extinction probability of a time homogeneous linear birth and death process under the influence of randomly occurring disasters. Math. Biosci. 164, 93-102.

Lindholm, M., 2008. On the time to extinction for a two-type version of Bartlett's epidemic model. Math. Biosci. 212, 99-108.

Nåsell, I., 1996. The quasi-stationary distribution of the closed endemic SIS model. Adv. Appl. Prob. 28, 895-932.

Nåsell, I., 1999a. On the quasi-stationary distribution of the stochastic logistic epidemic. Math. Biosci. 156, 21-40.

Nåsell, I., 1999b. On the time to extinction in recurrent epidemics. J. R. Statist. Soc. B 61, 309-330.

Nåsell, I., 2001. Extinction and quasi-stationarity in the Verhulst logistic model. J. Theor. Biol. 211, 11-27.

Nåsell, I., 2002. Stochastic models of some endemic infections. Math. Biosci. 179, 1-19.

Neuts, M.F, Li, J.M., 1996. An algorithmic study of $S-I-R$ stochastic epidemic models. In: Heyde, C.C., Prohorov, Yu V., Pyke, R., Rachev, S.T. (Eds.), Athens Conference on Applied Probability and Time Series, vol. 1. SpringerVerlag, Heidelberg, pp. 295-306.

Norden, R.H., 1982. On the distribution of the time to extinction in the stochastic logistic population model. Adv. Appl. Prob. 14, 687-708.

Pollett, P.K., 2001. Quasi-stationarity in populations that are subject to largescale mortality or emigration. Environ. Int. 27, 231-236.

Seneta, E., 1981. Non-Negative Matrices and Markov Chains. Springer, New York.

Stirk, E.R., Molina-París, C., van der Berg, H.A., 2008. Stochastic niche structure and diversity maintenance in the $\mathrm{T}$ cell repertoire. J. Theor. Biol. 255, 
237-249.

Swift, R.J., 2001. A logistic birth-death-immigration-emigration process. Math. Scientist 26, 25-33.

van Doorn, E.A., Pollett, P.K., 2008. Survival in a quasi-death process. Linear Algebra Appl. 429, 776-791.

van Herwaarden, O.A., Grasman, J., 1995. Stochastic epidemics: major outbreaks and the duration of the endemic period. J. Math. Biol. 33, 581-601.

$\mathrm{Xu}$, Y., Allen, L.J.S., Perelson, A.S., 2007. Stochastic model of an influenza epidemic with drug resistance. J. Theor. Biol. 248, 179-193. 